\title{
Demonstration of a quantum-enhanced fiber Sagnac interferometer
}

\author{
Moritz Mehmet, ${ }^{1,2}$ Tobias Eberle, ${ }^{1,2}$ Sebastian Steinlechner, ${ }^{1}$ Henning Vahlbruch, ${ }^{1}$ and Roman Schnabel ${ }^{1, *}$ \\ ${ }^{1}$ Max-Planck-Institut für Gravitationsphysik (Albert-Einstein-Institut) and Institut für Gravitationsphysik der \\ Leibniz Universität Hannover, Callinstrasse 38, 30167 Hannover, Germany \\ ${ }^{2}$ Centre for Quantum Engineering and Space-Time Research-QUEST, Leibniz Universität Hannover, Welfengarten 1 , \\ 30167 Hannover, Germany \\ *Corresponding author: Roman.Schnabel@aei.mpg.de
}

Received March 1, 2010; accepted April 8, 2010;

posted April 21, 2010 (Doc. ID 124858); published May 10, 2010

\begin{abstract}
The injection of squeezed light can be used to improve the sensitivity of an interferometer beyond the limit imposed by the zero-point fluctuation of the electromagnetic field. Here, we report on the realization of such a quantum-enhanced interferometer with a fiber-based Sagnac topology. Continuous wave squeezed states at $1550 \mathrm{~nm}$ with a noise reduction of $6.4 \mathrm{~dB}$ below shot noise were produced by type I optical parametric amplification and subsequently injected into the dark port of the interferometer. A reduction of the interferometer shot noise by $4.5 \mathrm{~dB}$ was observed, and the enhancement of the signal-to-noise ratio for a phase modulation signal generated within the interferometer could be demonstrated. We achieved a $95 \%$ fiber transmission for the squeezed states, which suggests that corresponding fiber-based quantum metrology and communication systems are feasible. (C) 2010 Optical Society of America
\end{abstract}

OCIS codes: $270.0270,270.6570,120.3180$.

Fiber Sagnac interferometers are used as optical rotation sensors and fiber optic gyroscopes [1], but their versatility has led to numerous further applications that include, among others, fiber loop mirrors [2] serving as high reflectors, fiber optic current sensors [3], and applications in the automotive industry [4].

For a given geometry and laser power, the sensitivity of any such optical measurement device is fundamentally limited by the so-called shot noise. For uncorrelated photons, i.e., for coherent states of light, the smallest measurable phase change is given by $\Delta \phi \geqslant 1 / \sqrt{N}$, where $N$ is the photon number per measurement time. Consequently, the sensors' performance can be improved by increasing the laser power. However, excess phase and polarization noise stemming from photon-phonon interactions like stimulated Raman scattering [5], stimulated Brillouin scattering, and guided acoustic-wave Brillouin scattering [6] constrain the maximum transmittable power through conventional all-silica fibers. An arbitrary increase in laser power to improve the sensitivity is therefore not possible.

In 1981, Caves [7] envisaged a way to enable the measurement beyond the shot-noise limit (SNL). The quantum-mechanical approach to the interferometer response (to a phase signal) showed that all the measurement noise comes from only one quadrature of the vacuum field entering the interferometer through the unused port of its 50:50 beam splitter. Caves' idea was to replace the vacuum field by a squeezed field that exhibits less noise in this quadrature, thereby reducing the quantum noise and hence increasing the signal to shot noise ratio. The two quadrature components of the electromagnetic field are the amplitude and phase quadrature, $\hat{X}_{1}$ and $\hat{X}_{2}$, respectively. Their variances are restricted by an uncertainty relation of the form $\Delta^{2} \hat{X}_{1} \cdot \Delta^{2} \hat{X}_{2} \geqslant 1$. The minimum uncertainty state with symmetrically distributed noise $\Delta^{2} \hat{X}_{1}$ $=\Delta^{2} \hat{X}_{2}=1$ corresponds to the field's ground state and the so-called vacuum field. A state with a quadrature variance below unity is called a squeezed state, and the variance suppression factor is called the squeezing factor. Following Caves' suggestions several tabletop experiments demonstrated quantum enhanced Mach-Zehnder [8], polarization [9], and Michelson interferometers [10-12]. In all of these experiments freely propagating continuous-wave (cw) laser beams were used. Generally, a critical issue in the quantum enhancement of interferometers is the optical loss introduced by the interferometer, because loss is a decoherence effect and degrades the squeezing factor. A quantum enhanced fiber-based interferometer is more difficult to realize because of absorption and scattering in the optical fiber. Also, the mode matching of a squeezed field into a fiber is critical and should be close to perfect. The same is true for the quantum efficiency of the final photoelectric detection. For instance, an optical loss of $20 \%$ deminishes a squeezing factor from 10 to 3.6; see, for example [13]. In the pulsed-laser regime squeezed states of light are produced by using the optical Kerr effect in fibers. Unfortunately, the high laser intensities required set Raman-induced limits to efficient squeezing in optical fibers [5]. Squeezed states of cw laser radiation are efficiently produced by parametric downconversion in optical resonators containing a second-order nonlinear crystal. Squeezing factors greater than ten have been realized recently at the laser wavelength of $1064 \mathrm{~nm}[13,14]$. Also recently, squeezed states of $\mathrm{cw}$ laser light at $1550 \mathrm{~nm}$ could be produced [15]. This wavelength is in the c-band transmission window of standard telecommunication fibers, where attenuation and dispersion effects are comparatively weak. 
In this Letter, we report the realization of a fiber Sagnac interferometer with a sensitivity beyond its SNL. Squeezed light from a nonlinear resonator was injected into the fiber interferometer. The interferometer signal and the squeezed quantum noise were detected by a high-quantum-efficiency balanced homodyne detector. A nonclassical sensitivity improvement of $4.5 \mathrm{~dB}$ was achieved. Figure 1 illustrates our experimental setup. A $10 \mathrm{~m}$ fiber Sagnac interferometer was operated with a cw erbium microfiber laser at $1550 \mathrm{~nm}$. The two output fields of the 50:50 Sagnac beam splitter (SBS) were carefully mode matched into the ends of a $10 \mathrm{~m}$ polarization maintaining PANDA-type fiber with $10.5 \mu \mathrm{m}$ mode field diameter and numerical aperture of 0.12 . In addition to two lenses in each arm of the interferometer, aspheric lenses with $f=11 \mathrm{~mm}$ were used to compensate for the strong divergence due to the fiber. The fiber had antireflective (AR) coatings on its end faces to minimize coupling loss for the traversing light. The single-path transmission efficiency was determined to be $95( \pm 1) \%$ by a relative power measurement with a single photodetector in front of and behind the fiber.

For the generation of squeezed light at $1550 \mathrm{~nm}$ we employed type I parametric downconversion (optical parametric amplification) in a $2 \mathrm{~mm} \times 1 \mathrm{~mm}$ $\times 10 \mathrm{~mm}$ periodically poled potassium titanyl phosphate (PPKTP) crystal inside a single-ended standing wave cavity with coupling-mirror power reflectivity of $90 \%$ and $20 \%$ for the fundamental and

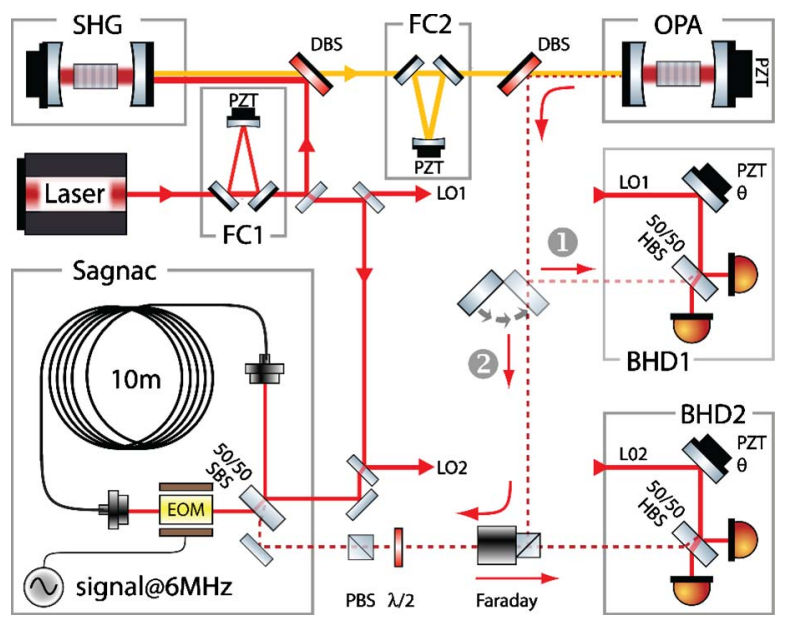

Fig. 1. (Color online) Schematic diagram of the optical setup. The experiment is driven by a fiber laser emitting $1550 \mathrm{~nm}$ radiation. SHG, second-harmonic generator producing the $775 \mathrm{~nm}$ pump field; FCs, filter cavities for spatial mode cleaning; PZT, piezoelectric transducer; DBS, dichroic beam splitter; OPA, optical parametric amplifier generating squeezed states; LO, local oscillator beam of the balanced homodyne detector, BHD. For characterization the squeezed field was sent via path 1 onto BHD1. To quantum enhance the Sagnac interferometer the squeezing was sent along path 2 and was injected into the dark signal port by using a Faraday rotator, half-wave plate, and a polarizing beam splitter (PBS). EOM, electro-optic modulator to generate a phase modulation signal inside the Sagnac interferometer. The interferometer signal and the squeezed quantum noise were detected with BHD2. harmonic field, respectively. The crystal had AR coatings on its end faces with residual reflection $\approx 0.25 \%$, which together with $0.1 \% / \mathrm{cm}$ absorption loss within the crystal and the mirror transmittance of $10 \%$ resulted in $90 \%$ escape efficiency. The second-harmonic pump field at $775 \mathrm{~nm}$ was generated in another nonlinear standing wave cavity. For details on the squeezed light generation at $1550 \mathrm{~nm}$ we refer to [15]. The squeezed field from the optical parametric amplifier (OPA) cavity was guided by a flip mirror into a balanced homodyne detector (BHD1) for characterization (path 1 in Fig. 1). The visibility with the homodyning local oscillator (LO) was 0.994. By changing the relative phase $\theta$ between the LO and the signal beam with a piezo actuated mirror the measured quadrature $X(\theta)=\cos (\theta) X_{1}+\sin (\theta) X_{2}$ was determined. The collected data are shown in Fig. 2. The vacuum (shot) noise reference level was measured with the signal input blocked. When the signal port was opened and the phase angle was set to detect the squeezed quadrature $\left[X(0)=X_{1}\right]$, a noise reduction of $6.4 \mathrm{~dB}$ below the shot noise was obtained. By switching to the orthogonal quadrature $[X(\pi / 2)$ $=X_{2}$ ] the corresponding antisqueezing of $11.1 \mathrm{~dB}$ above shot noise was recorded. These results can be well explained by a simple model assuming $16 \%$ optical loss, zero phase noise, and an initially pure state with $11.8 \mathrm{~dB}$ of squeezing (solid lines in Fig. 2). In our experiment photons were lost owing to the imperfect escape efficiency $[10( \pm 3) \%]$, during propagation $[1( \pm 0.5) \%]$, imperfect homodyne visibility $(1.2 \%)$, and due to the limited quantum efficiency of our custom-made photodiodes, which we infer to be about $96( \pm 3) \%$. Obviously the measured squeezing strength was primarily limited by loss due to the imperfect AR coatings on the PPKTP crystal itself. After the characterization of the field produced by the OPA the squeezed mode was guided via path 2 (Fig. 1) and was precisely modematched to the (dark) signal port mode of the Sagnac interferometer. To generate a phase-modulation signal inside the interferometer a

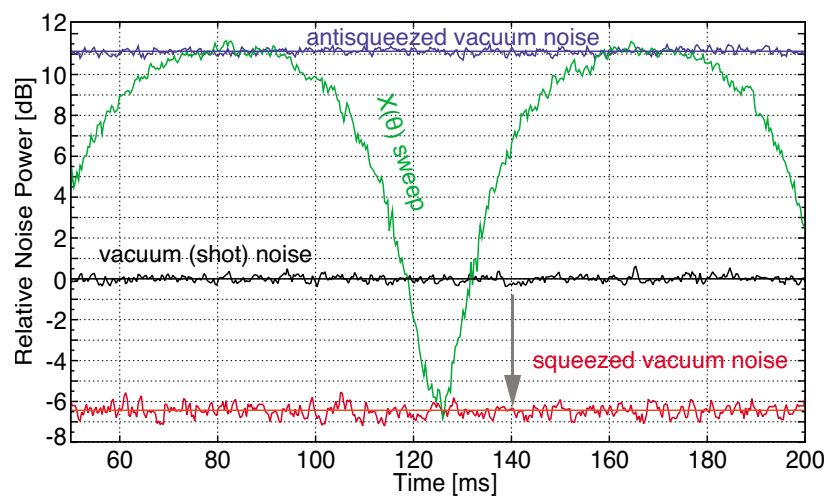

Fig. 2. (Color online) Noise powers of the squeezed states initially emitted by the OPA at a sideband frequency of $5 \mathrm{MHz}$ normalized to the shot-noise level. Shot-noise, squeezed noise, and antisqueezed noise were averaged twice. $X(\theta)$ was recorded by linearly sweeping the LO phase. All traces were recorded with a resolution bandwidth of $300 \mathrm{kHz}$ and a video bandwidth of $300 \mathrm{~Hz}$; the detector dark noise (20 dB below shot noise) was not subtracted. 
broadband electro-optic modulator (EOM) that could be driven by a signal generator was placed close to the Sagnac beam splitter. The signal at the output port was analyzed with a second balanced homodyne detector (BHD2). Figure 3 shows a $6 \mathrm{MHz}$ phase modulation measured with the Sagnac interferometer without (a) and with (b) squeezed light input. Both traces were normalized to the vacuum and recorded with a resolution bandwidth of $200 \mathrm{kHz}$ and a video bandwidth of $200 \mathrm{~Hz}$. The curves were averaged twice; the dark noise was not subtracted from the data. The Sagnac input power was $200 \mu \mathrm{W}$, and the LO power $1.75 \mathrm{~mW}$, which yielded a dark noise clearance of about $16 \mathrm{~dB}$. The LO phase was set to the signal maximum; the phase of the pump and the length of the OPA cavity were set to yield maximum squeezing. By the injection of squeezed vacuum a noise reduction of $4.5 \mathrm{~dB}$ below shot noise was achieved, which corresponds to a coherent state laser power increase of 2.8 and a phase sensitivity increase by a factor of about 1.7. When the squeezing was injected into the interferometer further optical loss occurred, which limited the nonclassical sensitivity improvement. The assumption of an additional 15\% loss could account for this degradation. The detection efficiencies for BHD1 and BHD2 were almost equivalent and did not need to be taken into account. Independent measurements provided about $2.5( \pm 1) \%$ loss due to the imperfect overlap between the squeezed field and the Sagnac, $4( \pm 1) \%$ loss due to the double pass through the Faraday rotator, PBSs, and a waveplate, $1.2 \%$ due to the contrast at the Sagnac beam splitter, and $5( \pm 1) \%$ due to the transmission through the fiber. In addition to that we assume $2( \pm 1) \%$ propagation loss inside the interferometer due to the lenses and the EOM and 0.5\% loss due to lenses and mirrors needed for the squeezed light input. These contributions yield an additional loss of approximately $14( \pm 1) \%$ which, given the experimental uncertainties, accounts well for our observations. Note

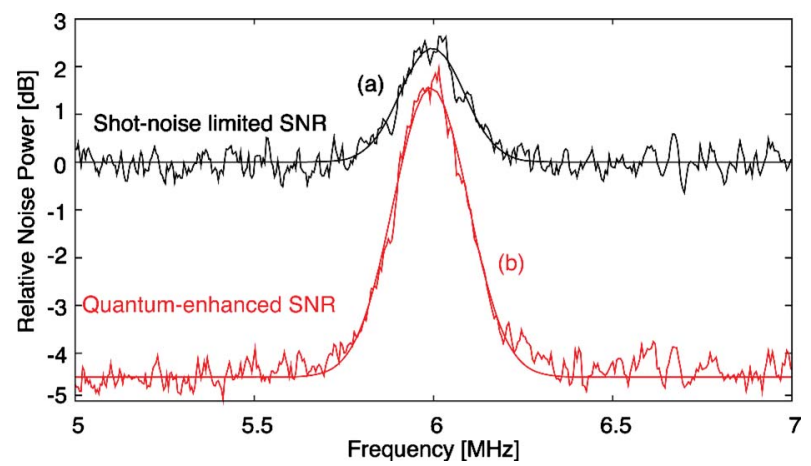

Fig. 3. (Color online) Sagnac interferometer output signal as recorded by the balanced homodyne detector. A $6 \mathrm{MHz}$ phase modulation was measured without [trace (a)] and with [trace (b)] squeezed light input, which yielded a reduction of the interferometer shot noise by $4.5 \mathrm{~dB}$. that light exiting the fiber (both directions) could be matched to a reference cavity with an overlap of 0.996 and 0.997 , respectively, illustrating that the transmission efficiency of $95 \%$ was essentially limited by suboptimal AR coatings.

In conclusion, we have demonstrated the nonclassical sensitivity improvement of a $10 \mathrm{~m}$ fiber Sagnac interferometer by $4.5 \mathrm{~dB}$ beyond its SNL. The total loss due to the input mode matching into the fiber and due to the output mode matching to a spatially filtered local oscillator beam was just 5\%. Given that conventional optical fibers can have losses as low as $\sim 0.2 \mathrm{~dB} / \mathrm{km}$ [16], the $6.4 \mathrm{~dB}$ squeezed states generated here could be transmitted over a fiber length of $8 \mathrm{~km}$ and would still show $3 \mathrm{~dB}$ of squeezing, assuming a 5\% loss due to input-output mode matching. Our results show that the quantum enhancement of local area (kilometer-size) optical fiber networks and fiber-based measurement devices with squeezed light are feasible.

The authors acknowledge the Deutsche Forschungsgemeinschaft (DFG) and the Centre for Quantum Engineering and Space-Time Research QUEST for financial support.

\section{References}

1. B. Culshaw, Meas. Sci. Technol. 17, R1 (2006).

2. D. Mortimore, J. Lightwave Technol. 6, 1217 (1988).

3. J. Blake, P. Tantaswadi, and R. de Carvalho, IEEE Trans. Power Deliv. 11, 116 (1996).

4. J. M. López-Higuera, Handbook of Optical Fibre Sensing Technology (Wiley, 2002).

5. R. Dong, J. Heersink, J. F. Corney, P. D. Drummond, U. L. Andersen, and G. Leuchs, Opt. Lett. 33, 116 (2008).

6. D. Elser, C. Wittmann, U. L. Andersen, O. Glockl, S. Lorenz, C. Marquardt, and G. Leuchs, J. Phys.: Conf. Ser. 92, 012108 (2007).

7. C. M. Caves, Phys. Rev. D 23, 1693 (1981).

8. M. Xiao, L.-A. Wu, and H. J. Kimble, Phys. Rev. Lett. 59, 278 (1987).

9. P. Grangier, R. E. Slusher, B. Yurke, and A. LaPorta, Phys. Rev. Lett. 59, 2153 (1987).

10. K. McKenzie, D. A. Shaddock, D. E. McClelland, B. C. Buchler, and P. K. Lam, Phys. Rev. Lett. 88, 231102 (2002).

11. H. Vahlbruch, S. Chelkowski, B. Hage, A. Franzen, K. Danzmann, and R. Schnabel, Phys. Rev. Lett. 95, 211102 (2005).

12. K. Goda, O. Miyakawa, E. E. Mikhailov, S. Saraf, R. Adhikari, K. McKenzie, R. Ward, S. Vass, A. J. Weinstein, and N. Mavalvala, Nat. Phys. 4, 472 (2008).

13. H. Vahlbruch, M. Mehmet, S. Chelkowski, B. Hage, A. Franzen, N. Lastzka, S. Goßler, K. Danzmann, and R. Schnabel, Phys. Rev. Lett. 100, 033602 (2008).

14. M. Mehmet, H. Vahlbruch, N. Lastzka, K. Danzmann, and R. Schnabel, Phys. Rev. A 81, 013814 (2010).

15. M. Mehmet, S. Steinlechner, T. Eberle, H. Vahlbruch, A. Thüring, K. Danzmann, and R. Schnabel, Opt. Lett. 34, 1060 (2009).

16. M.-J. Li and D. Nolan, J. Lightwave Technol. 26, 1079 (2008) 